\title{
Remarks on the Surface Area and Equality Conditions in Regular Forms Part II: Quadratic Prisms
}

\author{
Ahmed A. Elkhateeb • Esraa A. Elkhateeb
}

Published online: 27 June 2014

(C) Kim Williams Books, Turin 2014

\begin{abstract}
Following the same methodology and rules that were previously applied in Part I of this work, this part presents the remarks of the mathematical analysis for the regular quadratic right prisms. These include the rectangular and isosceles trapezoidal rooms. The first remark examines the effect of $\theta$ (or $\theta$ and $\beta$ ) on $\mathrm{S}$. The second remark calculates the minimum total surface area $\left(\mathrm{S}_{\text {Min }}\right)$ in two cases, case of constant $\theta$ (or $\theta$ and $\beta$ ) and case of variable $\theta$ (or $\theta$ and/or $\beta$ ). The third remark calculates the two ratios $\mathrm{R}_{\mathrm{W}}$ and $\boldsymbol{R}_{\boldsymbol{W o}}$. The last remark studies the required conditions for the numerical equality between (Per-Ar), and (S-V).
\end{abstract}

Keywords Trigonometry - Algebra - Differential equations · Volume · Area $\cdot$ Total surface area $\cdot$ Perimeter $\cdot$ Regular polygons $\cdot$ Right quadratic prisms · Minimum total surface area $\cdot$ Walls ratio $\cdot$ Numerical equality

\section{Introduction}

In the first part of this work (Elkhateeb 2014), assumptions were set out to mathematically analyze isosceles triangular right prisms in order to answer five questions:

- How the angle $\theta$ (or $\theta$ and $\beta$ ) affects $S$ ?

- When $\mathrm{S}$ becomes minimum $\left(\mathrm{S}_{\mathrm{Min}}\right)$ ?

\footnotetext{
A. A. Elkhateeb ( ( )

Department of Architecture, Faculty of Environmental Designs, King Abdulaziz University, Jeddah, Saudi Arabia

e-mail: aelkhateeb@kau.edu.sa

E. A. Elkhateeb

Physics Department, Faculty of Science, Ain Shams University, Cairo, Egypt

e-mail: dr.esraaelkhateeb@gmail.com
} 
- What is the ratio between walls surface area $S_{\mathrm{W}}$ and $S\left(S_{\mathrm{W}} / S=R_{\mathrm{W}}\right)$ ?

- When Ar numerically equals Per? and,

- When S numerically equals V?

Applying the same methodology and assumptions that were previously established in Part I, this part investigates the case of regular quadratic right prisms. The bases of such prisms could be either rectangular or isosceles trapezoidal, both will be considered in this part.

\section{Notations}

In this part, the following terms mean:

Ar : Room floor area $\left(\mathrm{m}^{2}\right)$

$\mathrm{h} \quad$ : The diagonal of the rectangle or trapezoid $(\mathrm{m})$

$\boldsymbol{h}_{\mathbf{0}}$ : The critical diagonal, the diagonal that fulfills (Per-Ar) equality (m)

$\mathrm{H}_{\mathrm{R}} \quad$ : Room height, the height of the prism (m)

$\boldsymbol{H}_{\boldsymbol{R} \boldsymbol{o}}$ : The critical room height, the height that fulfills (S-V) equality (m)

Per : Perimeter $(\mathrm{m})$

$\mathrm{S} \quad$ : Room total surface area $\left(\mathrm{m}^{2}\right)$

$\mathrm{S}_{\text {Min }}:$ The minimum total surface area $\left(\mathrm{m}^{2}\right)$

$\mathrm{S}_{\mathrm{W}}$ : Walls total surface area $\left(\mathrm{m}^{2}\right)$

$\mathrm{R}_{\mathrm{W}}$ : Walls ratio, $\mathrm{S}_{\mathrm{W}} / \mathrm{S}$ (Ratio)

$\boldsymbol{R}_{\boldsymbol{W o}}$ : The critical walls ratio, the ratio between walls total surface area and total surface area when $\mathrm{S}$ is minimum $\left(\mathrm{S}_{\mathrm{Min}}\right)$ (Ratio)

$\mathrm{V} \quad$ : Room volume $\left(\mathrm{m}^{3}\right)$

$\omega_{o}:$ The critical ratio, the ratio between $H_{R}$ and $r$ when $S$ is minimum $\left(S_{\text {Min }}\right)$ (Ratio)

The other terms will be illustrated in figures according to each case as required.

\section{Rectangular Rooms}

The rectangular rooms are the most common rooms in architectural applications. There is almost no building that doesn't contain a rectangular room. Figure 1 identifies the terms: $\theta, \psi, \mathrm{a}, \mathrm{b}, \mathrm{h}$ and $\mathrm{H}_{\mathrm{R}}$ in the rectangular room. During this part, it is assumed that the angle $\theta, \mathrm{Ar}$ and $\mathrm{V}$ are the independent variables whereas Per, and $\mathrm{S}$ are the dependent ones.

The Mathematical Relationships of Rectangular Prisms

Similar to the isosceles triangle, a rectangle can be completely identified knowing both $\mathrm{Ar}$ and $\theta$. This section derives the main mathematical functions among $\theta, \mathrm{h}$, Per, S and V (see Fig. 1). These functions will be utilized later to determine $S_{\text {Min }}$ and calculate the equality conditions. From the first principles, it can be proved that: 


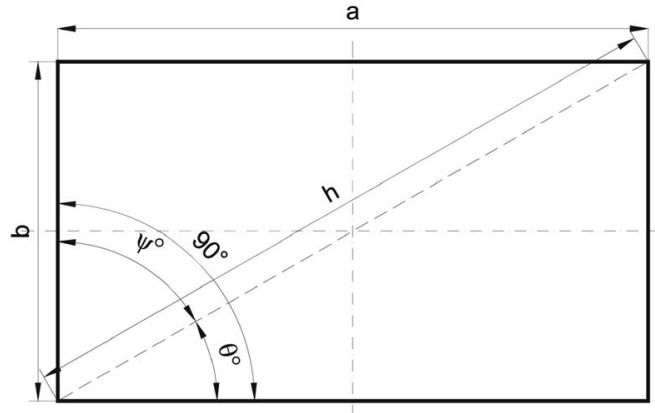

(a)

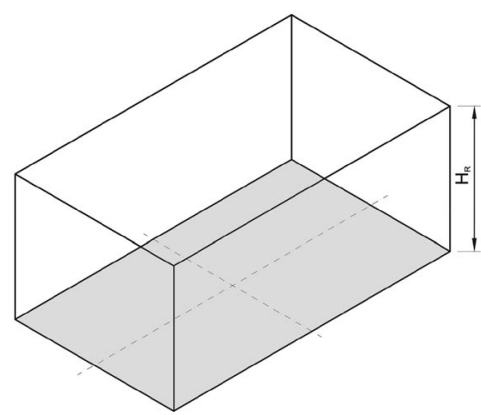

(b)

Fig. 1 Rectangular rooms, the different variables. a Left room plan; b right room 3-D

$$
a=\sqrt{\frac{A r}{\tan \theta}}
$$

Also

$$
a=h \cos \theta
$$

Thus

$$
\boldsymbol{b}=\boldsymbol{a} \tan \boldsymbol{\theta} .
$$

From Eq. 3, the perimeter Per $(2 a+2 b)$ can be calculated as:

$$
\boldsymbol{P e r}=2 \boldsymbol{a}(1+\tan \boldsymbol{\theta}) .
$$

By substitution for a from Eq. 1, Per can be also calculated as:

$$
\boldsymbol{P e r}=2 \sqrt{\frac{\boldsymbol{A r}}{\tan \boldsymbol{\theta}}}(1+\tan \boldsymbol{\theta}) \text {. }
$$

From Eq. 1, Ar can be calculated as:

$$
\boldsymbol{A r}=\boldsymbol{a}^{2} \tan \boldsymbol{\theta}
$$

In the third dimension, a rectangular shape can be extruded to form a right prism. In this case, its volume $\mathrm{V}=\left(\mathrm{H}_{\mathrm{R}} \times \mathrm{Ar}\right)$ can be calculated from:

$$
\boldsymbol{V}=\boldsymbol{a}^{2} \boldsymbol{H}_{\boldsymbol{R}} \tan \boldsymbol{\theta}
$$

Thus

$$
H_{R}=\frac{V}{a^{2} \tan \theta} .
$$

The total surface area $\mathrm{S}$ of a right prism with rectangular bases can be calculated as:

$$
\boldsymbol{S}=2 \boldsymbol{A r}+\left(\boldsymbol{P e r} \times \boldsymbol{H}_{\boldsymbol{R}}\right) .
$$

Given the values of Per (Eq. 4), $\operatorname{Ar}$ (Eq. 6), and $\mathrm{H}_{\mathrm{R}}$ (Eq. 8) as a function of $\theta$, Eq. 9 can be rewritten as: 


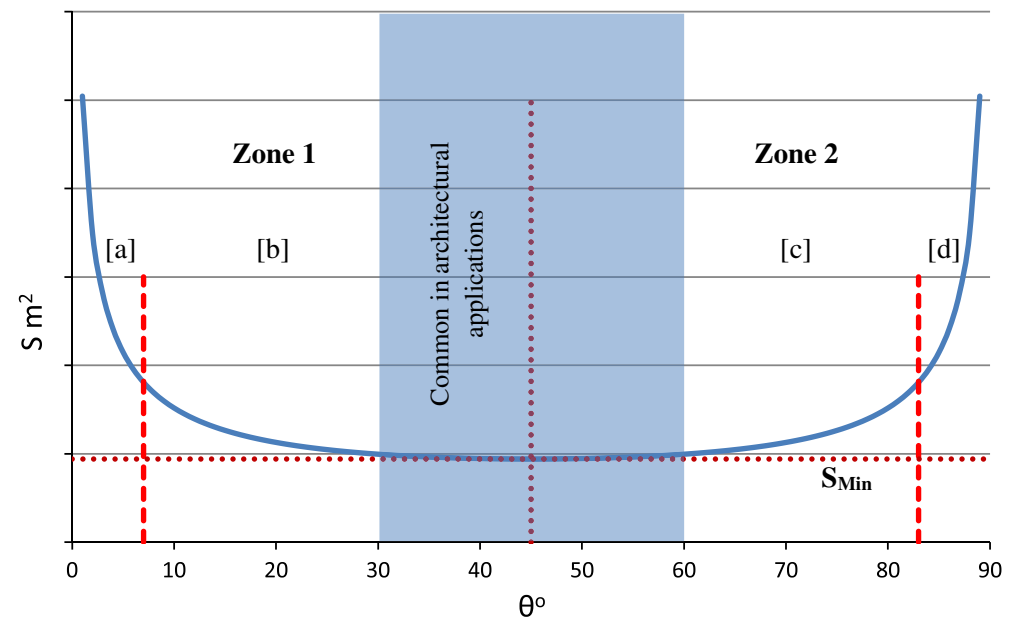

Fig. 2 The relationship between $\theta$ and $\mathrm{S}$ according to Eq. 10

$$
S=2 a^{2} \tan \theta+2 a H_{R}(1+\tan \theta)
$$

or

$$
\boldsymbol{S}=2 \boldsymbol{a}^{2} \tan \boldsymbol{\theta}+\frac{2 \boldsymbol{V}}{\boldsymbol{a} \tan \boldsymbol{\theta}}(1+\tan \boldsymbol{\theta}) .
$$

\section{Remark 1: Effect of $\theta$ on $S$}

Generally, the relationship between $\theta$ and $\mathrm{S}$ in the case of the rectangular rooms resembles that of the triangular rooms. Figure 2 is a graphical representation for Eq. 10. As can be concluded from this figure, the function is symmetrical around a vertical axis that passes through $\theta=45^{\circ}$. This has been expected earlier provided that the room is rectangular (i.e., $\theta+\psi=90^{\circ}$ ). The function reaches its minimum value at $\theta=45^{\circ}$. Again, this angle $\left(45^{\circ}\right)$ splits the function into two main zones:

- Zone 1: This zone encloses between $0^{\circ}<\theta \leq 45^{\circ}$, in this zone $\mathrm{S}$ is a decreasing function of $\theta$. This zone can be also divided into two sub-zones:

- Zone of rapid decay [a] $\left(0^{\circ}<\theta \leq 7^{\circ}\right)$ : where $\mathrm{S}$ loses more than $40 \%$ of its maximum value.

- Zone of slow decay $[\mathrm{b}]\left(7^{\circ} \leq \theta \leq 45^{\circ}\right)$ : $\theta$ increases rapidly in comparison with the reduction in $\mathrm{S}$ (in this zone, $\mathrm{S}$ loses about $10 \%$ of its value at $\left.\theta=7^{\circ}\right)$.

- Zone 2: in this zone $\mathrm{S}$ is an increasing function of $\theta$. This zone (between $45^{\circ} \leq \theta<90^{\circ}$ ) can be also divided into two additional sub-zones [c] (up to $\theta \leq 83^{\circ}$ ), and [d]. Both zones are similar to the sub-zones [b] and [a] respectively. 
Remark 2: the Minimum Total Surface Area, $S_{\text {Min }}$

Following the same approach previously applied in case of triangular rooms, two cases will be considered:

- Case of constant $\theta$, where both Ar and $\mathrm{H}_{\mathrm{R}}$ will be variables, or

- Case of variable $\theta$, where both $\mathrm{Ar}$ and $\mathrm{H}_{\mathrm{R}}$ will be constants.

Case I, Constant $\theta$, Variable $\mathrm{Ar}$ and $\mathrm{H}_{\mathrm{R}}$

In this case, among the different rectangular rooms that have the same $\theta$ and $\mathrm{V}, \mathrm{S}_{\mathrm{Min}}$ occurs when the first derivative of Eq. 11 equals zero, i.e.,

$$
\frac{d S}{d a}=4 a \tan \theta-\frac{2 V}{a^{2} \tan \theta}(1+\tan \theta)=0 .
$$

By Substitution for a and V according to Eqs. 2 and 7 respectively, Eq. 12 can be rewritten as:

$$
4 \boldsymbol{h} \cos \theta \tan \theta=\frac{2 \boldsymbol{a}^{2} \boldsymbol{H}_{\boldsymbol{R}} \tan \boldsymbol{\theta}}{\boldsymbol{a}^{2} \tan \boldsymbol{\theta}}(1+\tan \boldsymbol{\theta}) .
$$

By applying the rules of algebra and trigonometry, the critical ratio $\omega_{o}$ (see Sect. Notations) can be calculated from Eq. 13 as:

$$
\omega_{o}=\frac{2 \sin \theta}{(1+\tan \theta)} .
$$

Equation 14 indicates the condition under which $\mathrm{S}$ will reach its minimum value in a rectangular right prism. It is clear from Eq. 14 that $\omega_{o}$ in this case also depends entirely on $\theta$. To determine room dimensions that fulfill $\mathrm{S}_{\mathrm{Min}}$ :

- Determine both $\theta$ and V;

- Calculate $\omega_{o}$ by applying Eq. 14;

- Apply Eq. 8 to get h;

- Apply Eq. 14 again to get $\mathrm{H}_{\mathrm{R}}$;

- Utilize Eqs. 2 and 6 respectively to get a and Ar.

Figure 3 represents Eq. 14 and also the values of $\omega_{o}$ in the range $10^{\circ} \leq \theta \leq 80^{\circ}$. It can be concluded from this figure also that the function is symmetrical around $\theta=45^{\circ}$. In the range $\theta<45^{\circ}, \omega_{o}$ is an increasing function of $\theta$, whereas in the range $\theta>45^{\circ}, \omega_{o}$ is a decreasing function of $\theta$.

Again, the two relationships $\mathrm{H}_{\mathrm{R}}-\mathrm{Ar}$ on the one hand and $\mathrm{Ar}-\mathrm{S}$ on the other resemble the same relationships in case of the triangular rooms. Both relationships depend totally on $\omega_{o}$. As can be seen in Figs. 4 and $5, \omega_{o}$ divides the functions into two zones:

- Zone [a]: where $\omega<\omega_{\boldsymbol{o}}$. In this zone, $\mathrm{S}$ is a decreasing function of $\mathrm{H}_{\mathrm{R}}$ (see Fig. 4) and an increasing function of Ar (see Fig. 5), note that the location of the zones is reversed in Fig. 5. Thus, any increase in room height will decrease its total surface area. 


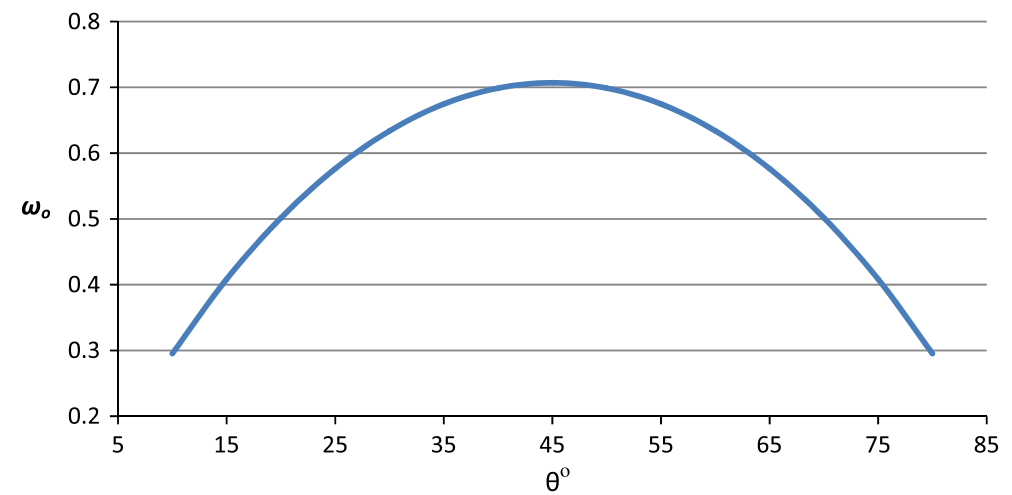

Fig. 3 Values of $\omega_{o}$ in the range $20^{\circ} \leq \theta \leq 80^{\circ}$ according to Eq. 14

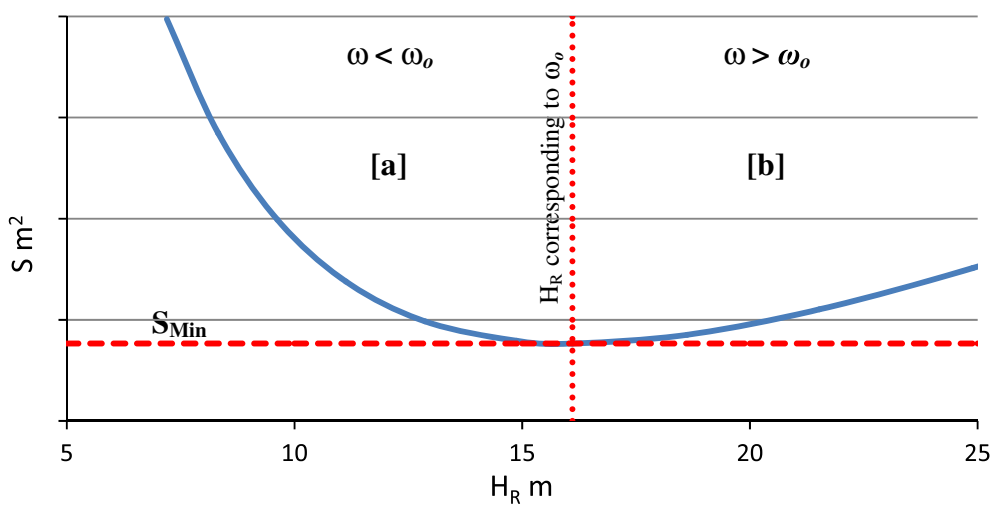

Fig. 4 The relationship of $\mathrm{H}_{\mathrm{R}}$ to $\mathrm{S}$ (case of $\theta=30^{\circ}$ )

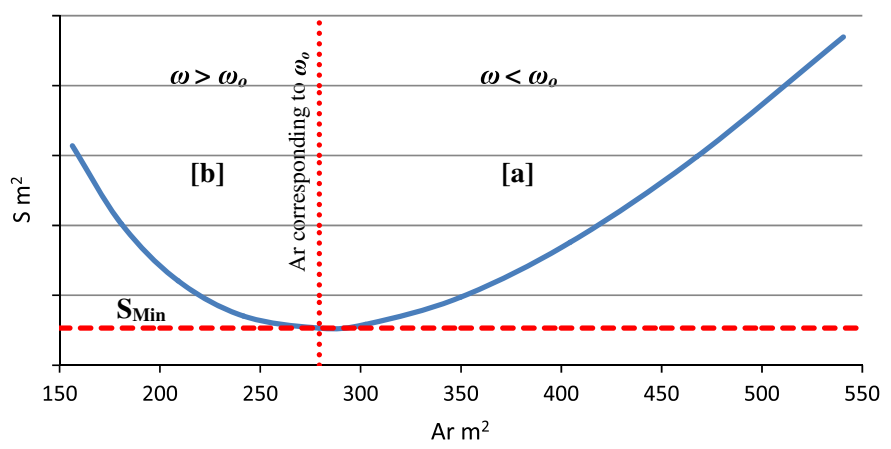

Fig. 5 The relationship of Ar to $\mathrm{S}$ (case of $\theta=30^{\circ}$ ) 
- Zone [b]: where $\omega>\omega_{\boldsymbol{o}}$. In this zone, $S$ is an increasing function of $\mathrm{H}_{\mathrm{R}}$ and a decreasing function of Ar. This means that, unlike zone [a], an increase in $\mathrm{H}_{R}$ will increase $\mathrm{S}$.

Case II, Variable $\theta$, Constant $\mathrm{Ar}$ and $\mathrm{H}_{\mathrm{R}}$

In this case, $\mathrm{V}, \mathrm{Ar}$ and $\mathrm{H}_{\mathrm{R}}$ are constants in all rooms, thus and according to Eq. 9, the perimeter will control the values of $\mathrm{S}$. In this case, among the different rectangular rooms, $S_{\text {Min }}$ occurs when the perimeter of the room reaches its minimum value. This can be mathematically calculated when the first derivative of Eq. 5 equals zero, i.e.,

$$
\frac{d P e r}{d \theta}=2 \sqrt{\frac{A r}{\tan \theta}}\left(\sec ^{2} \theta\right)+2(1+\tan \theta) \times \frac{1}{2} \sqrt{\frac{\tan \theta}{A r}} \times \frac{-A r}{\sin ^{2} \theta}=0 .
$$

By applying the rules of algebra and trigonometry, Eq. 15 will be:

$$
\tan \boldsymbol{\theta}=1 \text {, thus } \boldsymbol{\theta}=\mathbf{4 5}^{\circ} \text {, i.e. square. }
$$

This means that a room with a squared plan possesses the minimum perimeter among the other rectangular plans. Consequently, such a room has the minimum total surface area among the other rooms that have the same Ar and V but different $\theta$. This result completely agrees with the findings of Sect. Remark 1 (see Fig. 2).

Remark 3: Walls Ratio $\mathrm{R}_{\mathrm{W}}$

In rectangular rooms, $\mathrm{R}_{\mathrm{W}}$ can be mathematically defined as:

$$
\boldsymbol{R}_{W}=\frac{\boldsymbol{P e r} \times \mathrm{H}_{R}}{2 \mathrm{Ar}+\operatorname{Per} \times \mathrm{H}_{R}} .
$$

By substitution for Per and Ar from Eqs. 4 and 6 respectively, Eq. 17 can be rewritten as:

$$
\boldsymbol{R}_{W}=\frac{\boldsymbol{H}_{\boldsymbol{R}}(1+\tan \boldsymbol{\theta})}{\boldsymbol{a} \tan \boldsymbol{\theta}+\boldsymbol{H}_{\boldsymbol{R}}(1+\tan \boldsymbol{\theta})} .
$$

The relationship between $R_{W}$ and $\theta$ resembles the relationship between $S$ and $\theta$ (see Fig. 2). Thus it is symmetrical around $\theta=45^{\circ}$. In the zone where $\theta<45^{\circ}, R_{W}$ is a decreasing function of $\theta$. In the zone where $\theta>45^{\circ}, R_{W}$ is an increasing function of $\theta . R_{W}$ reaches its minimum value at $\theta=45^{\circ}$. To calculate $\boldsymbol{R}_{W o}$, the conditions for $\omega_{o}$ must be applied, thus, Eq. 18 can be rewritten as:

$$
\boldsymbol{R}_{\boldsymbol{W o}}=\frac{\frac{2 \boldsymbol{h} \sin \theta}{(1+\tan \theta)} \times(1+\tan \boldsymbol{\theta})}{\boldsymbol{h} \cos \boldsymbol{\theta} \tan \boldsymbol{\theta}+\frac{2 \boldsymbol{h} \sin \boldsymbol{\theta}}{(1+\tan \boldsymbol{\theta})} \times(1+\tan \boldsymbol{\theta})}
$$

This leads to:

$$
\boldsymbol{R}_{W o}=\frac{2}{3}=0.6667
$$


Thus, the critical walls ratio $\boldsymbol{R}_{W o}$ (see Sect. Notations) in rectangular right prisms is also constant for any $\theta$ and equals $2 / 3$. This is similar to isosceles triangular right prisms (Elkhateeb 2014).

\section{Remark 4: Case of Equality}

This section calculates two cases of numerical equality in rectangular rooms. The first considers the equality between Per and Ar. The last considers the equality between $\mathrm{S}$ and $\mathrm{V}$.

\section{Case I, Equality of Per and Ar}

In rectangular rooms and according to Eqs. 4 and 6, the numerical equality between Per and Ar occurs when:

$$
2 \boldsymbol{a}(1+\tan \theta)=\boldsymbol{a}^{2} \tan \boldsymbol{\theta} .
$$

By substitution for a from Eq. 2, and applying the rules of algebra and trigonometry, the critical diagonal $\boldsymbol{h}_{\boldsymbol{o}}$ (see Sect. Notations) can be calculated from Eq. 21 as:

$$
\boldsymbol{h}_{\boldsymbol{o}}=2(\sec \boldsymbol{\theta}+\csc \boldsymbol{\theta}) .
$$

Equation 22 indicates the condition for the numerical equality between Per and Ar. Likewise $\omega_{o}$, the equality in this case depends completely on $\theta$, for every $\theta$ there is a specific $\boldsymbol{h}_{\boldsymbol{o}}$ that fulfills this equality. The values of $\boldsymbol{h}_{\boldsymbol{o}}$ (in the range $20^{\circ} \leq \theta \leq 70^{\circ}$ ) were plotted in Fig. 6. As can be seen from this figure, the relationship between $\theta$ and $\boldsymbol{h}_{\boldsymbol{o}}$ is similar to the relationship between $\theta$ and $S$ (see Fig. 2), symmetrical around a vertical axis that passes through $\theta=45^{\circ}$. The values of other variables: a, b, Per and Ar can be calculated from Eqs. 2, 3, 4 and 6.

\section{Case II, Equality of S and V}

In this case, the numerical equality between $\mathrm{S}$ and $\mathrm{V}$ occurs when:

$$
2 \boldsymbol{A r}+\left(\boldsymbol{P e r} \times \boldsymbol{H}_{\boldsymbol{R o}}\right)=\boldsymbol{A r} \times \boldsymbol{H}_{\boldsymbol{R o}} .
$$

By substitution for Ar and Per according to Eqs. 2 and 4 respectively, and by applying the rules of algebra, Eq. 23 will be:

$$
\boldsymbol{H}_{\boldsymbol{R} \boldsymbol{o}}=\frac{2 \boldsymbol{a} \tan \boldsymbol{\theta}}{\boldsymbol{a} \tan \boldsymbol{\theta}-2(1+\tan \boldsymbol{\theta})} .
$$

If a was replaced by its equivalent value according to Eq. 2, and by applying the rules of trigonometry, Eq. 24 will be:

$$
\boldsymbol{H}_{\boldsymbol{R} \boldsymbol{o}}=\frac{2 \boldsymbol{h} \sin \boldsymbol{\theta}}{\boldsymbol{h} \sin \boldsymbol{\theta}-2(1+\tan \boldsymbol{\theta})} .
$$

For a rectangular room with given $\theta$ and $\mathrm{Ar}$, the numerical equality between $\mathrm{S}$ and $\mathrm{V}$ will occur if the condition of Eq. 24 (or 25) has been satisfied. This can be calculated in the following sequence: 


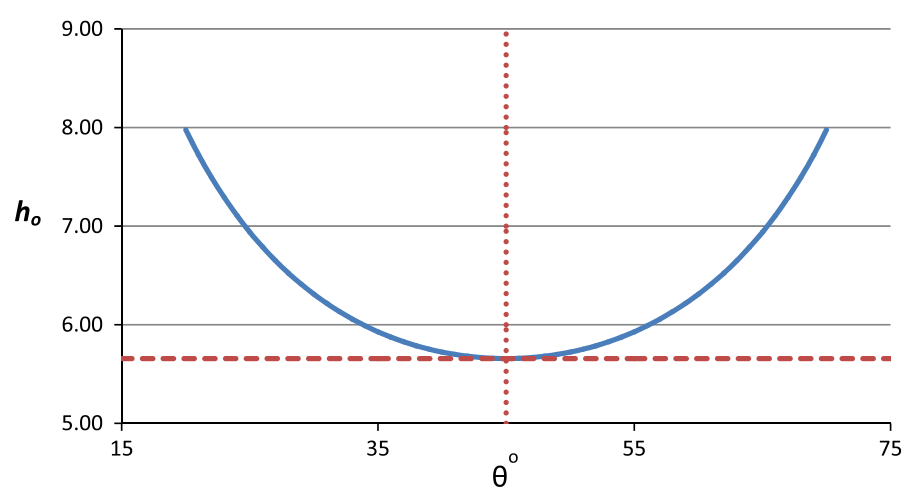

Fig. 6 Values of $h_{o}$ in the range $20^{\circ} \leq \theta \leq 70^{\circ}$ according to Eq. 22

- Determine both $\theta$ and Ar;

- Apply Eq. 1 to get a or Eq. 2 to get h, then;

- Substitute in Eq. 24 or 25, to get the critical room height $\boldsymbol{H}_{\boldsymbol{R} \boldsymbol{o}}$ (see Sect. Notations).

Similar to triangular rooms, the minus sign (-) in the denominator of Eq. 24 (or 25 ) indicates that for every $\theta$ there is a minimum a (accordingly $h$ ) under which this numerical equality will never exist. This occurs when $\boldsymbol{H}_{\boldsymbol{R} \boldsymbol{o}}$ tends to $\infty$, i.e., when $\mathrm{Ar}$ numerically equals Per according to Eq. 22. Figure 7 represents the relationship between $\mathrm{Ar}$ and $\boldsymbol{H}_{\boldsymbol{R} \boldsymbol{o}}$ calculated from Eq. 24 (for $\theta=30^{\circ}$ ). As can be seen from this figure, in the acceptable range, $\boldsymbol{H}_{\boldsymbol{R} \boldsymbol{o}}$ is a decreasing function of Ar while the function can be divided into two main zones, zone of rapid decay (when Ar tends to be equal to Per) and zone of slow decay (when Ar is far from this equality).

\section{Trapezoidal Rooms}

Trapezoidal, or fan-shaped, rooms are commonly used as auditoriums due to their good acoustic characteristics (Cremer et al. 1978). Dissimilar to triangular or rectangular shapes that can be mathematically identified knowing both their Ar and $\theta$, an isosceles trapezoidal shape needs more parameters in order to be completely identified. At least one of the following parameters $\mathrm{x}, \mathrm{a}, \mathrm{L}, \psi$ or $\beta$ (see Fig. 8) must be also given. In the following section it is assumed that the three parameters Ar, $\theta$ and $\beta$ are defined. $H_{R}$ is the height of the prism. During this section, it is also assumed that $\theta, \operatorname{Ar}, \beta$ and $\mathrm{V}$ are the independent variables whereas Per, and $\mathrm{S}$ are the dependent ones.

The Mathematical Relationships of Regular Trapezoidal Prisms

From the first principles, in an isosceles trapezoid it can be proved that:

$$
\boldsymbol{L}=\boldsymbol{h} \sin \theta
$$




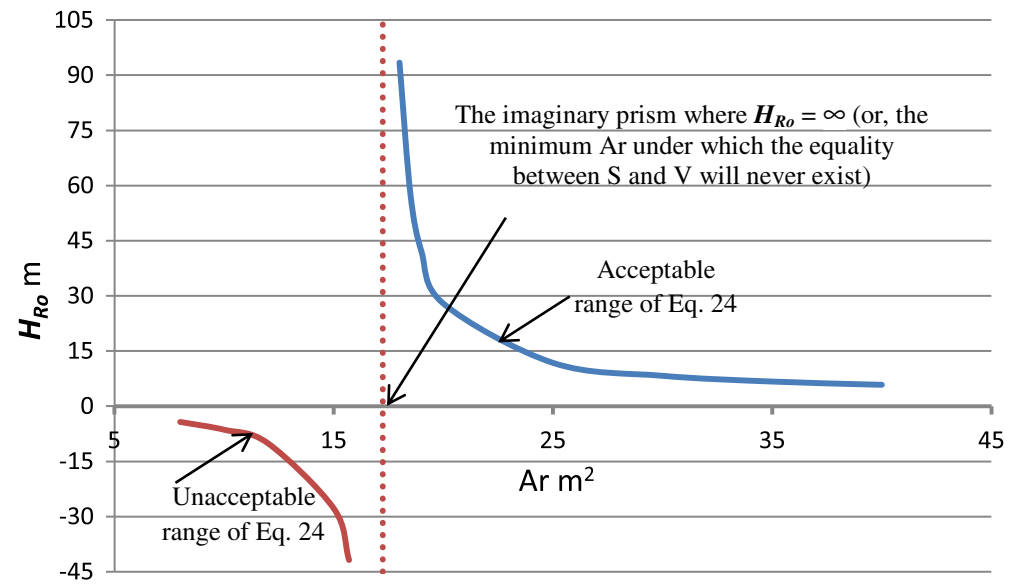

Fig. 7 The relationship of $\operatorname{Ar}$ to $\boldsymbol{H}_{\boldsymbol{R} o}$ (case of $\theta=30^{\circ}$ )

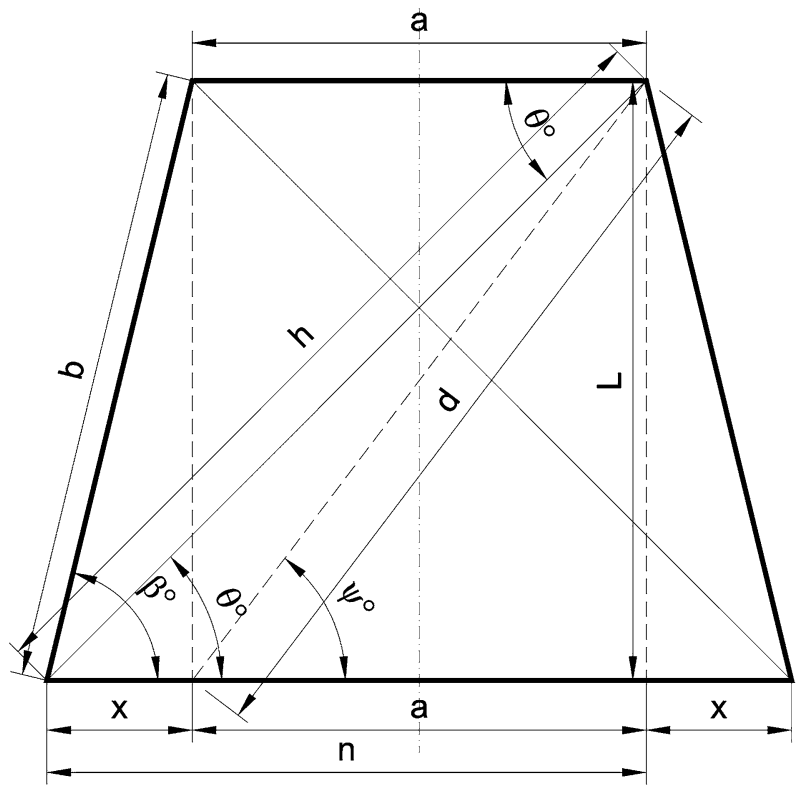

Fig. 8 Trapezoidal rooms, the different variables

$$
\begin{gathered}
n=\frac{A r}{L} \\
n=h \cos \theta \\
n=\sqrt{\frac{A r}{\tan \theta}}
\end{gathered}
$$




$$
\boldsymbol{b}=\frac{\boldsymbol{L}}{\sin \boldsymbol{\beta}} .
$$

By substitution for L according to Eqs. 26, Eq. 30 will be:

$$
\begin{aligned}
\boldsymbol{b} & =\frac{\boldsymbol{h} \sin \theta}{\sin \boldsymbol{\beta}} \\
\boldsymbol{h} & =\frac{\boldsymbol{n}}{\cos \theta}
\end{aligned}
$$

By substitution for $\mathrm{n}$ according to Eq. 29, and applying the rules of algebra and trigonometry, Eq. 32 will be:

$$
\boldsymbol{h}=\sqrt{\frac{A \boldsymbol{r}}{\sin \boldsymbol{\theta} \cos \theta}} .
$$

The perimeter Per of an isosceles trapezoid can be calculated from:

$$
\boldsymbol{P e r}=2(\boldsymbol{a}+\boldsymbol{x}+\boldsymbol{b})
$$

Thus, and according to Fig. 8, Eq. 34 can be rewritten as:

$$
\boldsymbol{P e r}=2(\boldsymbol{n}+\boldsymbol{b}) .
$$

The last equation can be set in different formats according to the values of both $\mathrm{n}$ and $\mathrm{b}$ as calculated in the previous equations. From Eqs. 28 and 31, Eq. 35 can be rewritten as:

$$
\boldsymbol{P e r}=2 \boldsymbol{h}\left(\cos \theta+\frac{\sin \theta}{\sin \beta}\right) .
$$

By substitution for $\mathrm{h}$ according to Eq. 33, Eq. 36 can be rewritten as:

$$
\boldsymbol{P e r}=2 \sqrt{\frac{A r}{\sin \theta \cos \theta}}\left(\cos \theta+\frac{\sin \theta}{\sin \beta}\right) .
$$

If $\mathrm{n}$ and $\mathrm{b}$ were replaced by their equivalent values according to Eqs. 27 and 30 respectively, Eq. 35 can be rewritten as:

$$
\boldsymbol{P e r}=2\left(\frac{\boldsymbol{A r}}{\boldsymbol{L}}+\frac{\boldsymbol{L}}{\sin \beta}\right) .
$$

If $\mathrm{L}$ was replaced by its equivalent value according to Eq. 26, the last formula will be:

$$
P e r=2\left(\frac{A r}{h \sin \theta}+\frac{h \sin \theta}{\sin \beta}\right)
$$

This leads to:

$$
\boldsymbol{P e r}=2\left(\frac{\boldsymbol{A r s i n} \beta+\boldsymbol{h}^{2} \sin ^{2} \theta}{\boldsymbol{h} \sin \theta \sin \beta}\right) .
$$

By substitution for $\mathrm{h}$ according to Eq. 33, Eq. 39 can be rewritten as: 


$$
\boldsymbol{P e r}=2\left[\frac{\left(A r \sin \beta+\frac{A r}{\sin \theta \cos \theta} \times \sin ^{2} \theta\right)}{\sqrt{\frac{A r}{\sin \theta \cos \theta}} \times \sin \theta \sin \beta}\right] .
$$

Applying the rules of algebra and trigonometry, Eq. 41 can be simplified to:

$$
\boldsymbol{P e r}=\frac{2 \sqrt{A r}}{\sin \beta}\left(\frac{\sin \beta+\tan \theta}{\sqrt{\tan \theta}}\right) .
$$

The area Ar of an isosceles trapezoid can be calculated from:

$$
\boldsymbol{A r}=\boldsymbol{L}(\boldsymbol{a}+\boldsymbol{x})
$$

Thus

$$
\boldsymbol{A r}=\boldsymbol{n} \boldsymbol{L} .
$$

By substitution for $\mathrm{L}$ and $\mathrm{n}$ according to Eqs. 26 and 28 respectively, Eq. 44 will be:

$$
\boldsymbol{A r}=\boldsymbol{h}^{2} \sin \boldsymbol{\theta} \cos \boldsymbol{\theta}
$$

In the third dimension and according to the assumptions, the isosceles trapezoidal plan will be extruded to form a right prism. Its volume $\left(\mathrm{Ar} \times \mathrm{H}_{\mathrm{R}}\right)$ can be calculated as:

$$
\boldsymbol{V}=\boldsymbol{h}^{2} \boldsymbol{H}_{\boldsymbol{R}} \sin \boldsymbol{\theta} \cos \boldsymbol{\theta}
$$

Thus, $\mathrm{H}_{\mathrm{R}}$ can be calculated as:

$$
H_{R}=\frac{V}{h^{2} \sin \theta \cos \theta} .
$$

The total surface area $\mathrm{S}$ of this prism can be calculated according to Eq. 9. By substitution for Per and Ar according to Eqs. 36 and 45 respectively, Eq. 9 will be:

$$
\boldsymbol{S}=2 \boldsymbol{h} \boldsymbol{H}_{\boldsymbol{R}}\left(\cos \theta+\frac{\sin \theta}{\sin \beta}\right)+2 \boldsymbol{h}^{2} \sin \theta \cos \theta .
$$

If $\mathrm{H}_{\mathrm{R}}$ was replaced by its equivalent value according to Eq. 47 , Eq. 48 can be rewritten as:

$$
S=\frac{2 \boldsymbol{V}}{\boldsymbol{h} \sin \theta \cos \theta}\left(\cos \theta+\frac{\sin \theta}{\sin \boldsymbol{\beta}}\right)+2 \boldsymbol{h}^{2} \sin \theta \cos \theta .
$$

\section{Remark 1: Effect of $\theta$ on $S$}

Figure 9 is a graphical representation for Eq. 48. As there are three independent variables in the isosceles trapezoid, the effect of $\theta$ on $\mathrm{S}$ is more complicated than the corresponding cases of triangle and rectangle. In case of the isosceles trapezoid, the minimum $\mathrm{S}$ depends on the three variables. In other words, there is $\mathrm{S}_{\text {Min }}$ for every combination of $\theta$ and $\beta$, this will be discussed in more details in Sect. Case II, variable $\theta$ and/or $\beta$. Nevertheless, the function resembles the case of triangular rooms, where the relationship is reversed when the function reaches its minimum 


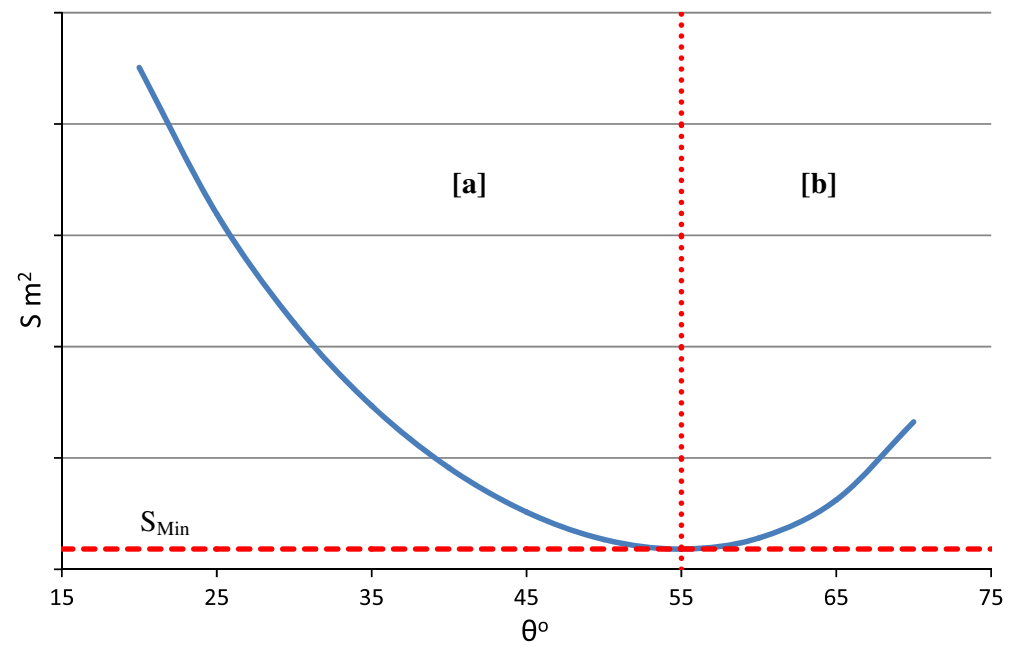

Fig. 9 The relationship between $\theta$ and $S$ according to Eq. 48 (both $\theta$ and $\beta$ are variables)

value, i.e., in Zone [a], $\mathrm{S}$ is a decreasing function of $\theta$, while in Zone [b], $\mathrm{S}$ is an increasing function of $\theta$ (see Fig. 9).

Remark 2: the Minimum Total Surface Area, $\mathrm{S}_{\mathrm{Min}}$

In isosceles trapezoidal right prisms, three independent variables ( $\mathrm{Ar}, \theta$ and $\beta$ ) yield more possibilities than the corresponding cases of triangular or rectangular prisms. In general, there are two main possibilities that may contain more sub-possibilities, they are:

- Case of constant $\theta$ and $\beta$, (variable Ar and $H_{R}$ )

- Case of variable $\theta$ and/or $\beta$, (constant Ar and $\mathrm{H}_{\mathrm{R}}$ ). This case can be divided into two additional sub-cases, these are:

- Case of constant $\theta$ and variable $\beta$;

- Case of variable $\theta$ and constant $\beta$.

Case I, Constant $\theta$ and $\beta$

In this case, both $h$ (i.e. Ar) and $H_{R}$ are variables whereas $\theta$ and $\beta$ are constants. Among the different isosceles trapezoidal right prisms that have the same $\theta, \beta$ and $\mathrm{V}, \mathrm{S}_{\mathrm{Min}}$ occurs when the first derivative of Eq. 49 equals zero, i.e.

$$
\frac{d S}{d h}=-\frac{2 V}{h^{2} \sin \theta \cos \theta}\left(\cos \theta+\frac{\sin \theta}{\sin \beta}\right)+4 h \sin \theta \cos \theta=0 .
$$

By substitution for $\mathrm{V}$ from Eq. 46, and by applying the rules of algebra and trigonometry, the critical ratio $\omega_{o}$ can be calculated from Eq. 50 as: 


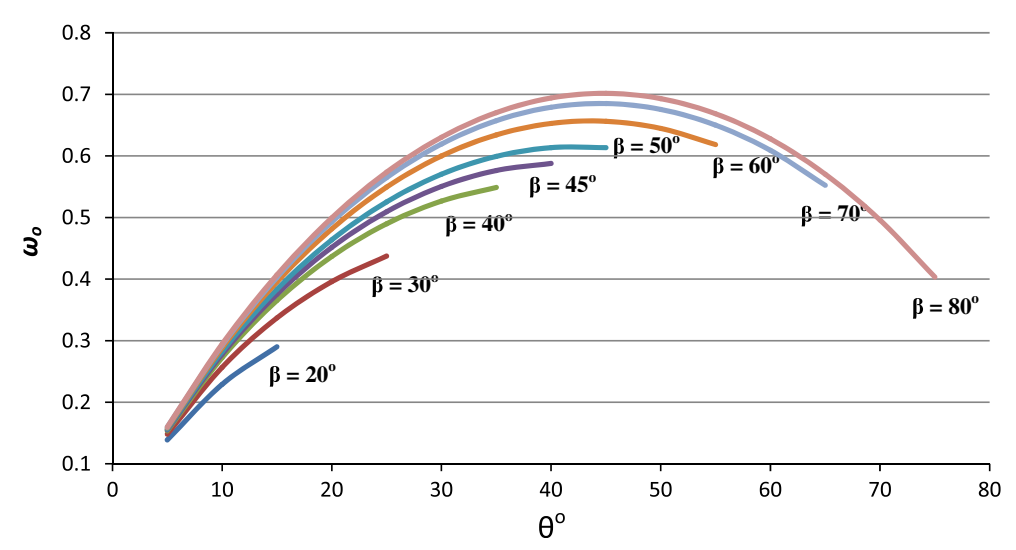

Fig. 10 Values of $\omega_{o}$ in the range $20^{\circ} \leq \beta \leq 80^{\circ}$ according to Eq. 51

$$
\omega_{o}=\frac{2 \sin \theta \cos \theta}{\cos \theta+\sin \theta \csc \beta} .
$$

Case I, Constant $\theta$ and $\beta$

By substitution in Eq. 47, h can be calculated. Knowing h, the other variables L, $\mathrm{n}$ and $\mathrm{b}$ can be calculated according to Eqs. 26, 28 and 31 respectively. Consequently the other parameters of the trapezoid can be calculated.

As can be concluded from Eq. $51, \omega_{o}$ is a function of both $\theta$ and $\beta$, i.e., for every combination of $\theta$ and $\beta(\theta<\beta)$ there is a value for $\omega_{o}$. This is obvious in Fig. 10, which is a graphical representation for Eq. 51 in the range $20^{\circ} \leq \beta \leq 80^{\circ}$ (values of $\omega_{o}$ calculated at $10^{\circ}$ intervals). It can be concluded from this figure that $\omega_{o}$ is an increasing function of $\theta$ as long as $\theta<45^{\circ}$. When $\theta$ exceeds $45^{\circ}, \omega_{o}$ is a decreasing function of $\theta$.

The relationships between $\left(\mathrm{H}_{\mathrm{R}}-\mathrm{S}\right)$ and $(\mathrm{Ar}-\mathrm{S})$ are similar to the same relationships in triangular and rectangular right prisms (see Figs. 4, 5), both depend entirely on $\omega_{o}$. In case $\omega<\omega_{o}$, $S$ is a decreasing function of $\mathrm{H}_{\mathrm{R}}$ and an increasing function of Ar. In case $\omega>\omega_{\boldsymbol{o}}, \mathrm{S}$ is an increasing function of $\mathrm{H}_{\mathrm{R}}$ and a decreasing function of Ar. Table 1 presents an example for an isosceles trapezoidal right prism where $\theta=15^{\circ}, \beta=20^{\circ}$ and $\mathrm{V}=4,500 \mathrm{~m}^{3}$. For the given prism, $\omega_{o}=0.29$ (see Fig. 10). $\mathrm{H}_{\mathrm{R}}$ is assumed for each case except for $\omega_{o}$ (the bold row in the table). As can be concluded from this table, the increase of $\mathrm{H}_{\mathrm{R}}$ in the zone $\omega>\omega_{\boldsymbol{o}}$ has a limited effect of $\mathrm{S}$ (around 0.1-10\% of $\mathrm{S}$ corresponding to $\omega_{o}$ ). Whereas the increase in Ar in the zone $\omega<\omega_{o}$ has a significant effect on S (around $0.1-28 \%$ of S corresponding to $\omega_{o}$ ).

Case II, Variable $\theta$ and/or $\beta$

In this case, both $\mathrm{Ar}$ and $\mathrm{H}_{\mathrm{R}}$ are similar to the cases of triangle and rectangle, $\mathrm{S}_{\mathrm{Min}}$ occurs when Per is minimum. As mentioned above, the case of variable $\theta$ and/or $\beta$ can be divided into two sub-cases, these are:

- Case of constant $\theta$ and variable $\beta$ 
Table 1 Example shows the effect of $\mathrm{Ar}$ and $\mathrm{H}_{\mathrm{R}}$ on $\mathrm{S}$ in isosceles trapezoidal right prisms (constant $\theta$ and $\beta$, and variable Ar)

\begin{tabular}{|c|c|c|c|c|c|c|c|c|c|c|c|c|}
\hline $\begin{array}{l}\theta \\
\left({ }^{\circ}\right)\end{array}$ & $\begin{array}{l}\beta \\
\left(^{\circ}\right)\end{array}$ & $\begin{array}{l}\mathrm{V} \\
\left(\mathrm{m}^{3}\right)\end{array}$ & $\boldsymbol{\omega}$ (ratio) & & $\mathrm{a}(\mathrm{m})$ & $\mathrm{b}(\mathrm{m})$ & $\mathrm{x}(\mathrm{m})$ & $\mathrm{h}(\mathrm{m})$ & $\operatorname{Ar}\left(m^{2}\right)$ & $\begin{array}{l}\mathrm{H}_{\mathrm{R}} \\
(\mathrm{m})\end{array}$ & $\mathrm{S}\left(\mathrm{m}^{2}\right)$ & $\begin{array}{l}\Delta \mathrm{S} \\
(\%)\end{array}$ \\
\hline \multirow[t]{18}{*}{15} & \multirow[t]{18}{*}{20} & \multirow[t]{18}{*}{4,500} & \multirow[t]{7}{*}{$\omega>\omega_{o}$} & 0.78 & 7.25 & 21.52 & 20.22 & 28.44 & 202.21 & 22.25 & $2,585.00$ & 10.0 \\
\hline & & & & 0.67 & 7.63 & 22.65 & 21.29 & 29.94 & 224.05 & 20.08 & $2,519.66$ & 7.2 \\
\hline & & & & 0.58 & 8.03 & 23.85 & 22.41 & 31.51 & 248.26 & 18.13 & $2,464.50$ & 4.9 \\
\hline & & & & 0.49 & 8.45 & 25.10 & 23.59 & 33.17 & 275.08 & 16.36 & $2,419.74$ & 3.0 \\
\hline & & & & 0.42 & 8.90 & 26.42 & 24.83 & 34.92 & 304.80 & 14.76 & $2,385.69$ & 1.5 \\
\hline & & & & 0.36 & 9.37 & 27.81 & 26.14 & 36.75 & 337.73 & 13.32 & $2,362.74$ & 0.5 \\
\hline & & & & 0.31 & 9.86 & 29.28 & 27.51 & 38.69 & 374.21 & 12.03 & $2,351.35$ & 0.1 \\
\hline & & & \multirow{11}{*}{$\begin{array}{l}\omega_{\boldsymbol{o}} \\
\omega<\omega_{o}\end{array}$} & 0.29 & 10.09 & 29.95 & 28.15 & 39.58 & 391.69 & 11.49 & $2,350.14$ & 0.0 \\
\hline & & & & 0.27 & 10.38 & 30.82 & 28.96 & 40.73 & 414.64 & 10.85 & $2,352.06$ & 0.1 \\
\hline & & & & 0.23 & 10.92 & 32.44 & 30.48 & 42.87 & 459.43 & 9.79 & $2,365.51$ & 0.7 \\
\hline & & & & 0.20 & 11.50 & 34.15 & 32.09 & 45.13 & 509.07 & 8.84 & $2,392.44$ & 1.8 \\
\hline & & & & 0.17 & 12.10 & 35.94 & 33.78 & 47.50 & 564.06 & 7.98 & $2,433.72$ & 3.6 \\
\hline & & & & 0.14 & 12.74 & 37.84 & 35.55 & 50.00 & 625.00 & 7.20 & $2,490.32$ & 6.0 \\
\hline & & & & 0.12 & 13.38 & 39.73 & 37.33 & 52.50 & 689.06 & 6.53 & $2,559.38$ & 8.9 \\
\hline & & & & 0.11 & 14.05 & 41.72 & 39.20 & 55.13 & 759.69 & 5.92 & $2,644.39$ & 12.5 \\
\hline & & & & 0.09 & 14.75 & 43.80 & 41.16 & 57.88 & 837.56 & 5.37 & $2,746.55$ & 16.9 \\
\hline & & & & 0.08 & 15.49 & 45.99 & 43.22 & 60.78 & 923.41 & 4.87 & $2,867.23$ & 22.0 \\
\hline & & & & 0.07 & 16.26 & 48.29 & 45.38 & 63.81 & $1,018.06$ & 4.42 & $3,007.94$ & 28.0 \\
\hline
\end{tabular}

- Case of variable $\theta$ and constant $\beta$

In the following sections, the condition(s) to fulfill $\mathrm{S}_{\mathrm{Min}}$ for each case will be addressed.

- Case of constant $\theta$ and variable $\beta$

In this case, to fulfill $\mathrm{S}_{\mathrm{Min}}$ the first derivative of Eq. 37 must be equal to zero, i.e.

$$
\frac{d P e r}{d \beta}=2 \sqrt{\frac{A r}{\sin \theta \cos \theta}} \times\left(-\frac{\sin \theta \cos \beta}{\sin ^{2} \beta}\right)=0
$$

Thus

$$
\frac{\sin \theta \cos \beta}{\sin ^{2} \beta}=0
$$

Consequently

$$
\cos \beta=0 \text {, i.e. } \beta=90^{\circ} .
$$

Equation 54 indicates that an isosceles trapezoidal right prism (with constant $\theta$ and variable $\beta$ ) will possess the minimum $S$ when $x \rightarrow 0$, i.e., the trapezoid tends to be a rectangle. In this case and according to Eq. 54, it is clear that $\mathrm{S}$ is a decreasing function of $\beta$ (see Fig. 11) regardless the value of $\theta$. It can be concluded also that $S$ 


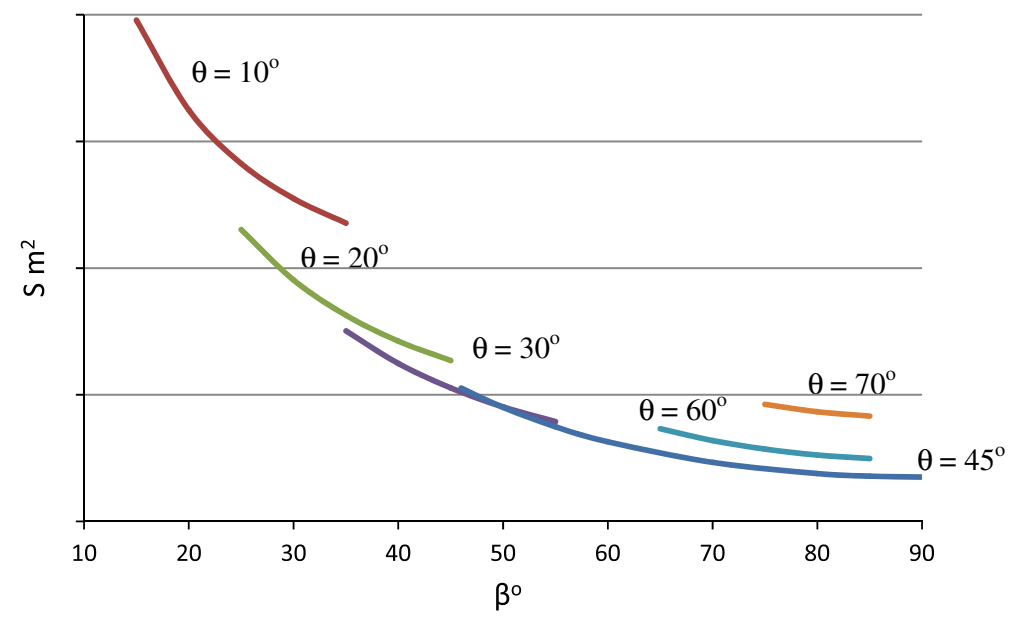

Fig. 11 The relationship between $\beta$ and $S$ (different $\theta$ )

is a decreasing function of $\theta$ in the range where $\theta \leq 45^{\circ}$. This relationship is reversed when $\theta>45^{\circ}$, i.e. $\mathrm{S}$ becomes an increasing function of $\theta$ (see Fig. 11) regardless the value of $\beta$.

- Case of variable $\theta$ and constant $\beta$

Unlike the previous case, here $\mathrm{L}$ is variable. Applying the rule of minimum Per, $\mathrm{S}_{\mathrm{Min}}$ occurs when the first derivative of Eq. 42 equals zero, i.e.,

$$
\frac{d P e r}{d \theta}=\frac{2 \sqrt{A r}}{\sin \beta} \times\left[\frac{-\sec ^{2} \theta}{2 \tan \theta \sqrt{\tan \theta}} \times\left(\sin \beta+\tan \theta+\frac{1}{\sqrt{\tan \theta}} \times \sec ^{2} \theta\right)\right]=0 \text {. }
$$

Applying the rules of trigonometry, this leads to:

$$
\sin \beta=\tan \theta
$$

Thus

$$
\theta=\tan ^{-1}(\sin \beta) .
$$

Consequently, among the different isosceles trapezoidal right prisms (with variable $\theta$ and constant $\beta$ ), the one that satisfies the condition of Eq. 57 possesses $S_{\text {Min }}$. Figure 12 shows an example for the relationship between $\theta$ and $S$ (case of $\beta=45^{\circ}$ ).

Equation 57 also reveals the results of the general case where both $\theta$ and $\beta$ are variables. In such cases, $S_{\text {Min }}$ exists when $\beta$ reaches its maximum value $\left(90^{\circ}\right)$. Thus, and according to Eq. 57, $\theta$ equals $45^{\circ}$. This means that $\theta=\psi$, or a squared shape. This result agrees with the findings of the rectangular prisms where a squared prism has the minimum $\mathrm{S}$ among other prisms that have the same Ar and V (see Sect. Case II, variable $\theta$, constant Ar and $H_{R}$ ).

Remark 3: Walls Ratio $\mathrm{R}_{\mathrm{W}}$

In isosceles trapezoidal right prisms, the ratio $\mathrm{R}_{\mathrm{W}}$ can be mathematically calculated from Eq. 17. By substitution for Per and Ar according to Eqs. 36 and 45 respectively, Eq. 17 will be: 


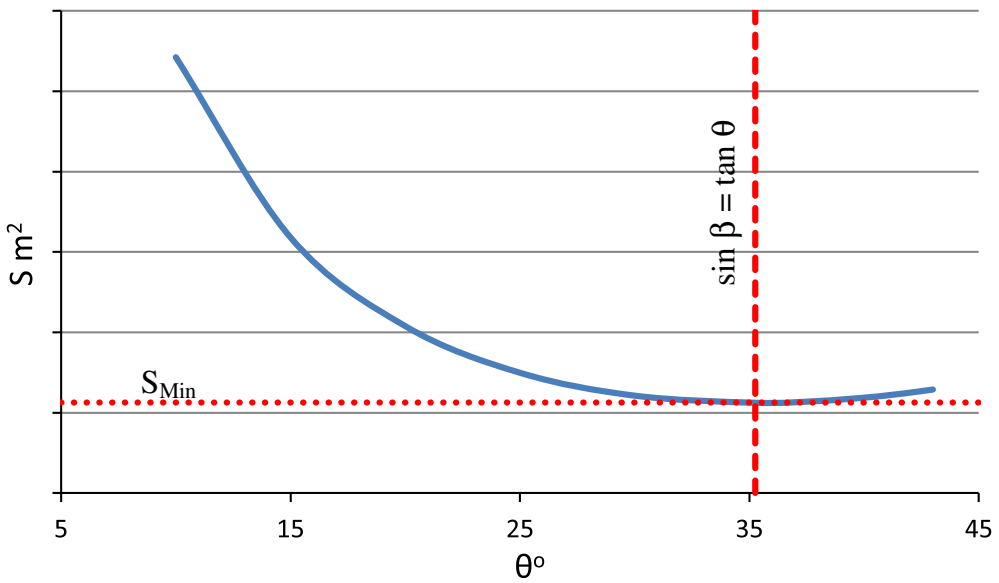

Fig. 12 The relationship between $\theta$ and $S$ (case of $\beta=45^{\circ}$ )

$$
\boldsymbol{R}_{W}=\frac{2 \boldsymbol{h} \boldsymbol{H}_{\boldsymbol{R}}\left(\cos \theta+\frac{\sin \theta}{\sin \beta}\right)}{2 \boldsymbol{h}^{2} \sin \theta \cos \theta+2 h H_{R}\left(\cos \theta+\frac{\sin \theta}{\sin \beta}\right)} .
$$

By applying the rules of algebra and trigonometry, Eq. 58 can be rewritten as:

$$
\boldsymbol{R}_{W}=\frac{H_{R}(\cos \theta+\sin \theta \csc \beta)}{h \sin \theta \cos \theta+H_{R}(\cos \theta+\sin \theta \csc \beta)} .
$$

To calculate $\boldsymbol{R}_{\boldsymbol{W}}$, the conditions for $\boldsymbol{\omega}_{\boldsymbol{o}}$ must be applied, thus, Eq. 58 will be written as:

$$
\boldsymbol{R}_{\boldsymbol{W o}}=\frac{4 \boldsymbol{h}^{2} \sin \boldsymbol{\theta} \cos \boldsymbol{\theta} \times\left(\frac{\left(\cos \theta+\frac{\sin \theta}{\sin \beta}\right)}{\left(\cos \theta+\frac{\sin \theta}{\sin \beta}\right)}\right)}{2 \boldsymbol{h}^{2} \sin \boldsymbol{\theta} \cos \boldsymbol{\theta}+4 \boldsymbol{h}^{2} \sin \boldsymbol{\theta} \cos \boldsymbol{\theta} \times\left(\frac{\left(\cos \theta+\frac{\sin \theta}{\sin \beta}\right)}{\left(\cos \theta+\frac{\sin \theta}{\sin \beta}\right)}\right)} .
$$

By applying the rules of trigonometry, this leads to:

$$
\boldsymbol{R}_{W o}=\frac{2}{3}=0.6667 \text {. }
$$

The last equation indicates that $\boldsymbol{R}_{\boldsymbol{W o}}$, similar to triangular and rectangular right prisms, is constant for any combination of $\theta$ and $\beta$ and equals $2 / 3$.

Remark 4: Case of Equality

Following the same methodology, this section calculates the two numerical equalities, (Per-Ar) and (S-V).

Case I, Equality of Per and Ar

In case of isosceles trapezoidal shapes, the critical diagonal $\boldsymbol{h}_{\boldsymbol{o}}$ (see Sect. Notations) that fulfills the numerical equality between Ar and Per can be calculated based on Eqs. 36 and 45 as: 


$$
2 h_{o}\left(\cos \theta+\frac{\sin \theta}{\sin \beta}\right)=h_{o}^{2} \sin \theta \cos \theta .
$$

By applying the rules of algebra and trigonometry, Eq. 62 can be simplified to:

$$
\boldsymbol{h}_{\boldsymbol{o}}=2(\csc \boldsymbol{\theta}+\sec \boldsymbol{\theta} \csc \boldsymbol{\beta}) \text {. }
$$

Equation 63 reveals the condition under which this numerical equality exists. Again, and similar to $\omega_{o}$, the numerical equality in this case is a function of both $\theta$ and $\beta$, for every combination of $\theta$ and $\beta$ there is a specific $\boldsymbol{h}_{\boldsymbol{o}}$ that fulfills this equality. Given the values of $\boldsymbol{h}_{\boldsymbol{o}}, \theta$ and $\beta$, the other parameters of a trapezoid: L, n, b, Per and Ar can be calculated from Eqs. 26, 28, 31, 36 and 45 respectively.

\section{Case II, Equality of S and V}

In isosceles trapezoidal right prisms, the critical room height $\boldsymbol{H}_{\boldsymbol{R}}$ (see Sect. Notations) that fulfills the numerical equality between $\mathrm{S}$ and $\mathrm{V}$ can be calculated from Eqs. 46 and 48 as:

$$
2 h^{2} \sin \theta \cos \theta+2 h H_{R o}\left(\cos \theta+\frac{\sin \theta}{\sin \beta}\right)=h^{2} \sin \theta \cos \theta \times H_{R o}
$$

By applying the rules of algebra and trigonometry, Eq. 64 can be simplified to:

$$
\boldsymbol{H}_{\boldsymbol{R} \boldsymbol{o}}=\frac{2 \boldsymbol{h} \sin \boldsymbol{\theta} \cos \boldsymbol{\theta}}{\boldsymbol{h} \sin \boldsymbol{\theta} \cos \boldsymbol{\theta}-2(\cos \boldsymbol{\theta}+\sin \boldsymbol{\theta} \csc \boldsymbol{\beta})} .
$$

For an isosceles trapezoidal room with given $\theta, \beta$ and $\mathrm{Ar}$, the numerical equality between $\mathrm{S}$ and $\mathrm{V}$ will exist if the condition in Eq. 65 fulfilled. This can be calculated in the following sequence:

- Determine $\theta, \beta$ and Ar;

- Apply Eq. 33 to get h, then;

- Substitute in Eq. 65, to get the critical room height $\boldsymbol{H}_{\boldsymbol{R} \boldsymbol{o}}$;

- Apply Eq. 46 to get V and Eq. 48 to get S.

Similar to the triangular and rectangular rooms, the minus sign (-) in the denominator of Eq. 65 indicates that for every $\theta$ and $\beta$ there is a minimum $\mathrm{h}$ under which this equality will never exist. Again, this occurs when $\boldsymbol{H}_{\boldsymbol{R} o}$ tends to $\infty$, i.e., when Ar equals Per according to Eq. 63. The relationship between Ar and $\boldsymbol{H}_{\boldsymbol{R} \boldsymbol{o}}$ resembles the same relationship in the case of the rectangular right prisms (see Fig. 7).

\section{Conclusions}

Following the same methodology and rules that were applied previously in Part I, this part examines the cases of the regular quadratic right prisms. Such prisms include the rectangular and isosceles trapezoidal right prisms; both were considered in this part. The first remark examines the effect of $\theta$ on S. In the second remark, the minimum total surface area $S_{\text {Min }}$ for the rooms under discussion was calculated in 
two cases, case of constant $\theta$ (or constant $\theta$ and $\beta$ ) and case of variable $\theta$ (or variable $\theta$ and/or $\beta$ ). In the first case, the critical ratio $\omega_{o}$ was calculated. Results showed that $\omega_{o}$ depends entirely on $\theta$ (or $\theta$ and $\beta$ in case of isosceles trapezoidal right prisms). The values of $\omega_{o}$ were calculated and presented. In the second case, where $\theta$ (or $\theta$ and/or $\beta$ in isosceles trapezoids) is variable, results showed that $S_{\text {Min }}$, in the case of rectangles, corresponds to $\theta=45^{\circ}$. In the case of trapezoids, results indicate that an isosceles trapezoidal right prism (with constant $\theta$ and variable $\beta$ ) will possess the minimum $S$ when $x \rightarrow 0$. In case of variable $\theta$ and constant $\beta$, the isosceles trapezoid that satisfies the condition of Eq. 57 possesses $S_{\text {Min }}$. The third remark calculates the ratio $R_{W}\left(S_{W} / S\right)$. In rectangles, results showed that $R_{W}$ reaches its minimum value when $\theta=45^{\circ}$ whereas in trapezoids, it depends on the values of $\theta$, $\beta$, h and $\mathrm{H}_{\mathrm{R}}$. Results also showed that the critical walls ratio $\boldsymbol{R}_{\boldsymbol{W o}}$ is constant for any $\theta$ (or $\theta$ and $\beta$ in isosceles trapezoids) and is equal to $2 / 3$. The last remark investigates the conditions for the numerical equality either between Per and Ar or S and $\mathrm{V}$. In the first case, the critical diagonal $\boldsymbol{h}_{\boldsymbol{o}}$ that fulfills Per-Ar equality was calculated. Results showed that $\boldsymbol{h}_{\boldsymbol{o}}$ depends entirely on $\theta$ (or $\theta$ and $\beta$ in isosceles trapezoids). In the second case, the critical room height $\boldsymbol{H}_{\boldsymbol{R}}$ that fulfills $\mathrm{S}-\mathrm{V}$ equality was calculated. Results also indicated that for every $\theta$ (or $\theta$ and $\beta$ in isosceles trapezoids) there is a minimum $\mathrm{h}$ under which this equality will never exist; this corresponds to $\boldsymbol{h}_{\boldsymbol{o}}$ (i.e. $\mathrm{Ar}=\mathrm{Per}$ ).

\title{
References
}

Cremer, L., and H. Müller. 1978. Principles and applications of room acoustics. Translated by Theodore J. Schultz. London: Applied science Publishers

Elkhateeb, A. 2014. Remarks on the surface area and equality conditions in regular forms, part Itriangular prisms. Nexus Network Journal, Architectural and Mathematics 16(1):219-232. doi:10. 1007/s00004-014-0178-8

\begin{abstract}
Ahmed Elkhateeb is an Egyptian architect who graduated in 1990 from the Department of Architecture, Faculty of Engineering of Ain Shams University (Cairo, Egypt). He worked in the same department until summer 2011. He completed his M.Sc. and Ph.D. in the field of architectural acoustics, which is his primary area of interest along with mathematics and its relation with architecture. He has many published researches in national and international scholarly journals in the field of building science, in addition to five published books in the same research area. He also supervised many theses in different areas related to architecture. He was awarded two international research Grants in the field of architectural acoustics. As of fall 2012 he is Full Professor of Architecture and Building Science in the Department of Architecture in the Faculty of Environmental Designs of King Abdulaziz University, Jeddah, Saudi Arabia.
\end{abstract}

Esraa A. Elkhateeb is an Egyptian Scientist graduated in 1986 from the Department of Physics, Faculty of Science of Ain Shams University (Cairo, Egypt). She worked in the same department. She completed her M.Sc. and Ph.D. in the field of theoretical physics. She has many published researches in national and international journals in Atomic, molecular, computational and high energy physics. 